\title{
Intra-session reliability of different angles of lateral wedge insoles in the biomechanical parameters of the knee
}

Vitor Ferreira ${ }^{1 *}$, Leandro Machado ${ }^{2}$, Rita Simões ${ }^{3}$ and Paulo Roriz ${ }^{4}$

*Correspondence: v.ferreira@ua.pt

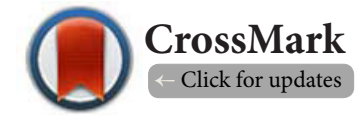

'School of Health Sciences, University of Aveiro (ESSUA), Portugal.

${ }^{2} \mathrm{CIF} 2 \mathrm{D}, \mathrm{LABIOMEP}$, Faculdade de Desporto da Universidade do Porto, Portugal.

${ }^{3}$ Santa Casa da Misericórdia da Mealhada, Portugal.

${ }^{4}$ CIDESD-ISMAI, LABIOMEP, Portugal.

\begin{abstract}
Background: Lateral wedged insoles are one of the biomechanical interventions recommended for individuals with medial knee osteoarthritis to reduce external knee adduction moment. Several approaches have already been tested to reduce the forces that crosses the knee. However, an approach based on individual biomechanical assessment of the acute effects of different angles of lateral wedge insoles is necessary.

Purpose: To assess the intra-session reliability of different angles of lateral wedge insoles on knee biomechanics during gait in healthy adults.

Methods: Twenty-five healthy volunteers (15 males, with mean age of $21.6 \pm 5.5$ years, body mass of $65.2 \pm 8.7 \mathrm{~kg}$, and height $169 \pm 7.5 \mathrm{~cm}$ ) were recruited. A Qualisys Oqus motion analysis system was used for kinematic and kinetic data collection during gait with the six experimental conditions: $0^{\circ}, 2^{\circ}, 4^{\circ}, 6^{\circ}, 8^{\circ}$ and 10-degree wedge insoles. The intraclass correlation (ICC) and their 95\% confidence intervals, the standard error of measurement (SEM) and the minimal detectable change (MDC) were calculated for each insole.

Results: For all kinematic parameters, ICC was superior to 0.924, showed an excellent consistency. For the kinetic parameters, the external knee abduction moment was the least consistent (ICC $>0.540)$. It was found that the estimated value for SEM was $0.014 \mathrm{Nm} / \mathrm{kg}$ for the first and second peak of external knee adduction moment with a corresponding MDC of $0.039 \mathrm{Nm} / \mathrm{kg}$ for both.
\end{abstract}

Conclusions: This study provides preliminary data which may be useful for clinical interpretation and decision to recommend the insole that best fits to the condition of the patient with medial knee osteoarthritis.

Keywords: External knee adduction moment, Gait analysis, Standard error of measurement, Minimal detectable change, Outcome assessment

\section{Introduction}

Lateral wedged insoles are one of the biomechanical interventions recommended for individuals with knee Osteoarthritis (OA) [1]. They have been associated with reduced external knee adduction moment (EKAM) [2-4]. The amount of wedging needed to induce a biomechanical response with clinical significance is an interesting field of research. Different amounts of lateral wedging have been proposed, such as $4[5], 5[4,6], 6[7], 10$ $[4,8]$ or 11 degrees [9]. So far, it seems not possible to reach a consensus on the correct amount of wedging [10]. However, results from previous studies could encourage an individual fitting, based on the perception that some individuals do not respond to a certain amount of wedging [11-13].

One way to assess the biomechanical effect of the insoles is to use three-dimensional gait analysis. This method is extensively used in clinical gait examination and research. The gait, although being a cyclical movement presents natural intrasubject variability, and can be attributed to many factors including inconsistent marker placement and anthropometric measurements, data processing and walking speed $[14,15]$. 
On the other hand, because no subject is equal to any other, the gait of different subjects, even if normal, also presents variability (i.e. inter-subject variability) [14]. It is this one that is affected by factors such as age, gender, weight, height, cadence, ...) and even by controlling these variables (same age, gender, height, weight, ...) it is expected a greater intervariability than intrasubject because the object (subject) is not the same. There is also the variability introduced by the measurement system and procedures and it is desired to be systematic and minimal [15]. That is, the instrument measures what it is supposed to measure, and the methods of acquisition are well-known. If the systematic error is high the instrument does not measure what it is supposed to measure, and its validity is compromised [16].

A correct adjustment of the wedge angle is desirable for each patient. A biomechanical assessment of the immediate effects may provide this adjustment. Testing different angles of wedging, first in healthy individuals, is the first step for individual fitting in individuals with knee OA. This involves an initial approach regarding the evaluation of the procedures to understand the intrarater reliability, the agreement and the minimal detectable change (MDC). The reliability may be defined as the ratio of variability between subjects to the total variability of all measurements in the sample [17], it is also known as an intraclass correlation (ICC), as it equals the correlation between any two measurements made on the same subject [18]. The agreement quantifies how close the scores for repeated measurements are and a popular way of quantifying it is to estimate the standard error of measurement (SEM) and the $95 \%$ limits of agreement [19]. MDC is described as the amount of change which is sufficiently greater than measurement error for the variable of interest [20].

To the best of our knowledge, the intra-session reliability of different angles of wedging during gait in knee biomechanics has never been tested. Understanding the ICC, SEM and MDC can be an important contribution to the individualized prescription of insoles. The selection of the most appropriate angle may improve the outcomes of the use of this type of insoles. The ability of clinicians to discern findings that are meaningful from those that are insignificant or artifactual is therefore essential particularly when the differences in insole angles are so small. Thus, the aim of this study was to assess the intra-session reliability of different angles of lateral wedge insole in kinematics and kinetics of the knee during gait in healthy adults.

\section{Methods \\ Participants}

Twenty-five healthy volunteers ( 15 males, with mean age of $21.6 \pm 5.5$ years old, body mass of $65.2 \pm 8.7 \mathrm{~kg}$, and height $169 \pm 7.5 \mathrm{~cm}$ ) were recruited. All subjects were screened by interview and physical examination. The inclusion criteria included healthy young adults, without any physical disability and aged from 20 to 40 years old. The participants were excluded based on any neurological or musculoskeletal disorder, particularly at the knee joint complex. The study was conducted following the principles of the Declaration of Helsinki and was approved by the Ethics Committee of the Faculty of Sport. All participants freely gave their written consent to participate in the study after a verbal and written explanation of the project.

\section{Equipment and data collect}

A motion analysis system (Qualisys, Gothenburg, Sweden) was used for kinematic and kinetic data acquisition. The setup included eleven three-dimensional cameras (Qualisys Oqus) working at a sampling frequency of $200 \mathrm{~Hz}$, and four force platforms (Bertec, Columbus, USA) with sampling frequency of $1000 \mathrm{~Hz}$. Participants were instructed to perform several walking trials along a 10-meter walkway. The Calibrated Anatomical System Technique (CAST) [21] was employed to characterize body segments motion during the walking trials. Reflective markers were attached bilaterally on the principal bony prominences of the subject's lower limbs and pelvis. The bony landmarks included the anterior superior iliac spine, the posterior superior iliac spine, the greater trochanter, the medial and lateral condyles, the fibula head, the tibial tubercle, and the lateral and medial malleolus. The markers located at the calcaneus, $1^{\text {st }}, 2^{\text {nd }}$ and $5^{\text {th }}$ metatarsal heads were attached to the shoes, and the feet were assumed as rigid bodies. Four rigid clusters were also placed bilaterally, on the anterior-lateral aspect of the thigh and of the lower-leg. All the markers remained attached to the body during the entire data collection. A previous static calibration of the markers was performed before walking trials to reconstruct the participant's biomechanical model, such as defining the joint center or defining the segments endpoints. A physiotherapist, with several years of experience, conducted the experiment, and participants were not given any walking instructions other than instructed to walk at their self-selected speed. Six valid trials, for each of the following six conditions were captured in a randomized order (using www.randomization.com): a control condition (shoes with a 0 -degree insole), and lateral wedge insoles with 2, 4, 6, 8 and 10 degrees, resulting in 36 valid trials per participant. Participants used their own shoes, and were allowed to perform a series of walking trials prior to data acquisition in order to adapt to the new insole condition. The insoles were custom-made with a long posterior pronating wedge (Capron Podologie, France; Ref.: 8004F). The insoles were used bilaterally to promote stability but only the data from the right side was used for analysis.

\section{Data analysis}

Post-processing calculation of the kinematic and kinetic time series data was conducted using Visual 3D software (Version 6.00.12, C-Motion, Rockville, USA). The kinematic and kinetic data were filtered $(6 \mathrm{~Hz})$ using a Butterworth $4^{\text {th }}$ order filter. Knee joint angles (using a XYZ Cardan sequence) and joint 
moments were computed and expressed relatively to the proximal segment. Joint moment was determined through inverse dynamics and stated as external moment. All variables were computed for the stance phase (normalized to 100\%) of the gait cycle. We chose only to analyze the stance phase because changes in the swing phase are not expected. Only peak values of the kinematic and kinetic data were analyzed. We separated the six valid trials per condition into two sets of 3 : set 1 - the first 3 trials; set 2 - the last 3 trials. A valid trial was set when the subject had walked naturally, and the entire foot had contacted the force plate surface without going beyond its contours. The peak value for each quantity was calculated as the mean of the three highest values found in each set, during the same session, for each of the six experimental conditions. In the case of EKAM two peaks have been retrieved from the EKAM waveform and used for analysis (named as first and second peak of the EKAM). The kinematic data were expressed in degrees $\left({ }^{\circ}\right)$. The kinetic data were normalized to body mass (BM) $(\mathrm{Nm} / \mathrm{kg})$. The knee adduction angular impulse (KAAI) was also calculated by integrating the EKAM signal for the stance phase (\% BM x Time).

The mean of the two sets of three trials were calculated for the main knee kinematic and kinetic parameters. The ICC and their $95 \%$ confidence intervals for the 2 -way mixed effects model (type: consistency) were calculated for key kinematic and kinetic parameters. Values for ICC $\leq 0.69$ were considered as poor, $0.70-0.79$ as fair, $0.80-0.89$ as good, and $\geq 0.90$ as excellent $[22,23]$. Calculations also included the mean difference (Diff) between the two sets of trials (set 1 minus set 2) and the $95 \% \mathrm{Cl}$ for Diff, the standard deviation of the differences (SDdiff) and the $95 \%$ limits of agreement $(95 \%$ LOA). The SEM and MDC were calculated using the following equations: $\mathrm{SEM}=\mathrm{SD} * \sqrt{ }(1-\mathrm{ICC})$ and $\mathrm{MDC}_{95}=1,96^{*} \sqrt{2}{ }^{*} \mathrm{SEM}[19]$. The ICC statistical analysis was conducted using SPSS (version 24.0; IBM, Chicago, IL).

\section{Results}

ICC, SEM, and MDC values for the session assessments of kinematic data are presented in Table 1. The kinematic parameters presented an excellent consistency along the trials. For all kinematic parameters ICC was superior to 0.924 (varying between 0.924 and 0.999 ). The mean Diff between sets of trials was inferior to $0.6^{\circ}$ in peak angles on the six insoles conditions. SEM between sets of trials were inferior to $1.0^{\circ}$ for all peak angles. MDC ranged from $0.3^{\circ}$ to $2.8^{\circ}$ in sagittal plane, $0.3^{\circ}$ to $0.9^{\circ}$ in coronal plane and $0.5^{\circ}$ to $2.9^{\circ}$ in transverse plane.

Table 2 displays ICC, SEM, and MDC values for the kinetic data. Most of the kinetic parameters showed good or excellent reliability with values ICC $>0.80$. However, peak abduction moment ( $4^{\circ}$ insole), peak extension moment ( $6^{\circ}$ insole) and peak abduction moment ( $10^{\circ}$ insole) show less consistency in the measurement.

Since the first peak of the EKAM does not necessarily correspond to the peak adduction of the knee in all individuals,
Table 3 shows the ICC, SEM and MDC values of the first and second peak of the EKAM, as well as the KAAI. EKAM parameters presented an excellent reliability on the six conditions under study: first peak of the EKAM shows an ICC $\geq 0.982$; second peak of the EKAM an ICC $\geq 0.978$ ) and KAAI an ICC $\geq 0.983$. SEM between sets of trials were inferior to $0.017 \mathrm{Nm} / \mathrm{kg}$ at the six conditions. The MDC in the first peak of the EKAM was less than $0.048 \mathrm{Nm} / \mathrm{kg}$.

When all conditions under study were pooled (average of insoles) (Table 3), it was found that the estimated value for SEM was $0.014 \mathrm{Nm} / \mathrm{kg}$ for the first and second peak of the EKAM with a corresponding MDC of $0.039 \mathrm{Nm} / \mathrm{kg}$ for both. The difference between set 1 and set 2 plotted against the mean of the sets indicated no obvious bias for the first peak of the EKAM (Figure 1), second peak of the EKAM (Figure 2) and KAAI (Figure 3).

\section{Discussion}

The primary purpose of this study was to assess the intrasession reliability of using lateral wedge insoles with different angles. The careful selection of the wedge angle that best fits the knee biomechanics is a challenge that may allow to improve the results of this type of device in patients with knee OA. Results indicated that the intra-session reliability were excellent for the main kinematic and kinetic parameters related to EKAM. The ICC is therefore considered a relative measure of reliability that provides an indication of how well a measure can differentiate between subjects [18]. An ICC of 0.988 for the average of insoles suggests that the first peak of the EKAM is appropriate for use. These findings are better than previous reliability reports that have evaluated healthy subjects. In the study of Wilken et al. [24], the ICC diverges in kinematics from 0.78 to 0.98 . However, the authors only described the values in the sagittal plane. Meldrum et al. [25]

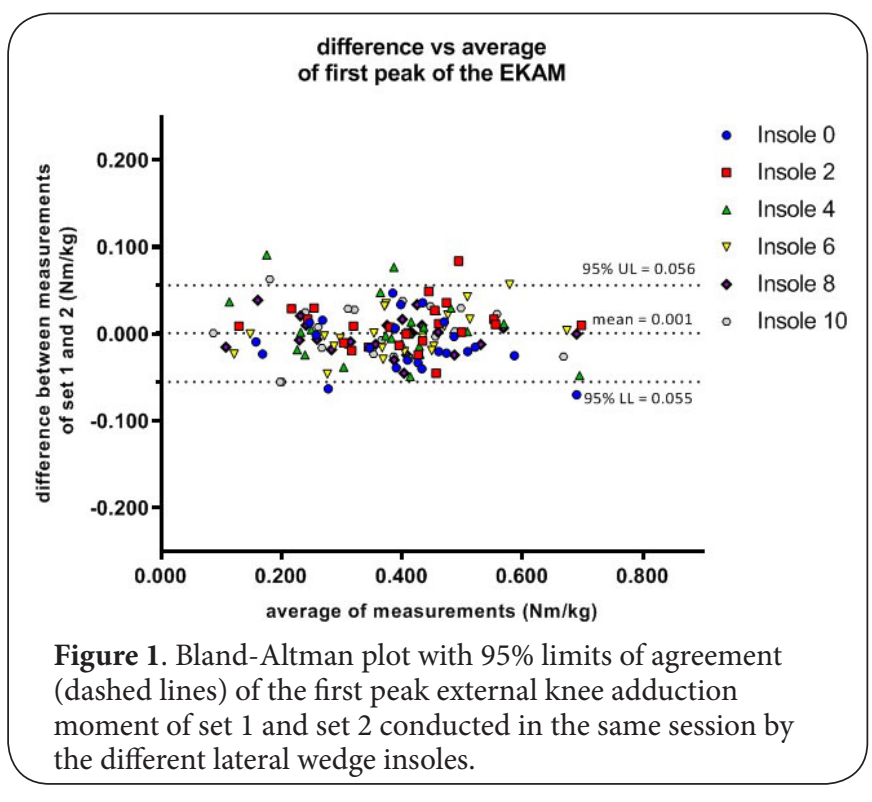


Ferreira et al., Physical Therapy and Rehabilitation 2020,

http://www.hoajonline.com/journals/pdf/2055-2386-7-10.pdf

doi: 10.7243/2055-2386-7-10

Table 1. Reliability values for knee kinematic parameters for different insoles: knee peak joint angle (stated in degrees, except for ICC and $95 \% \mathrm{CI})$.

\begin{tabular}{|c|c|c|c|c|c|c|c|c|c|c|c|c|}
\hline \multirow{2}{*}{$\begin{array}{l}\text { Kinematic }\left(^{\circ}\right) \\
0^{\circ} \text { Insole (Peak) }\end{array}$} & \multirow[t]{2}{*}{ ICC } & \multicolumn{2}{|c|}{$95 \% \mathrm{CI}$} & \multirow{2}{*}{ Mean } & \multirow[t]{2}{*}{ Minimum } & \multirow[t]{2}{*}{ Maximum } & \multirow[t]{2}{*}{ Diff } & \multirow[t]{2}{*}{$\mathrm{SD}_{\text {diff }}$} & \multirow[t]{2}{*}{ 95\% LL } & \multirow[t]{2}{*}{ 95\% UL } & \multirow[t]{2}{*}{ SEM } & \multirow[t]{2}{*}{ MDC } \\
\hline & & & & & & & & & & & & \\
\hline Flexion & 0,953 & 0,889 & 0,980 & 44,3 & 36,5 & 50,3 & 0,4 & 1,6 & $-2,7$ & 3,3 & 0,9 & 2,4 \\
\hline Extension & 0,951 & 0,884 & 0,979 & $-0,7$ & $-6,6$ & 7,2 & 0,0 & 1,4 & $-2,7$ & 2,7 & 0,7 & 1,9 \\
\hline Abduction & 0,991 & 0,978 & 0,996 & 1,7 & $-3,5$ & 10,2 & 0,0 & 0,3 & $-0,7$ & 0,9 & 0,3 & 0,9 \\
\hline Adduction & 0,990 & 0,976 & 0,996 & $-3,9$ & $-9,1$ & 3,5 & $-0,1$ & 0,5 & $-1,0$ & 1,1 & 0,3 & 0,9 \\
\hline Lateral rotation & 0,992 & 0,982 & 0,997 & $-15,6$ & $-30,0$ & $-4,9$ & $-0,2$ & 1,5 & $-3,1$ & 2,7 & 0,6 & 1,7 \\
\hline Medial rotation & 0,981 & 0,954 & 0,992 & $-7,6$ & $-23,7$ & 6,0 & 0,6 & 1,0 & $-1,3$ & 3,0 & 1,0 & 2,9 \\
\hline \multicolumn{13}{|l|}{$2^{\circ}$ Insole (Peak) } \\
\hline Flexion & 0,956 & 0,895 & 0,981 & 43,7 & 35,9 & 51,8 & 0,4 & 1,6 & $-2,6$ & 3,1 & 0,8 & 2,2 \\
\hline Extension & 0,972 & 0,935 & 0,988 & $-1,0$ & $-6,7$ & 6,9 & $-0,1$ & 1,0 & $-2,2$ & 2,1 & 0,5 & 1,5 \\
\hline Abduction & 0,999 & 0,998 & 1,000 & 1,7 & $-3,6$ & 10,0 & 0,0 & 0,2 & $-0,4$ & 0,4 & 0,1 & 0,3 \\
\hline Adduction & 0,997 & 0,992 & 0,999 & $-3,7$ & $-8,9$ & 3,3 & $-0,1$ & 0,4 & $-0,9$ & 0,7 & 0,2 & 0,7 \\
\hline Lateral rotation & 0,995 & 0,989 & 0,998 & $-16,0$ & $-29,8$ & $-5,1$ & $-0,2$ & 1,0 & $-2,1$ & 1,9 & 0,5 & 1,3 \\
\hline Medial rotation & 0,996 & 0,990 & 0,998 & $-7,4$ & $-23,3$ & 5,0 & $-0,4$ & 1,0 & $-2,3$ & 1,9 & 0,5 & 0,5 \\
\hline \multicolumn{13}{|l|}{$4^{\circ}$ Insole (Peak) } \\
\hline Flexion & 0,977 & 0,947 & 0,990 & 43,6 & 35,9 & 48,6 & 0,1 & 1,0 & $-2,0$ & 2,1 & 0,5 & 1,4 \\
\hline Extension & 0,977 & 0,945 & 0,990 & $-1,1$ & $-7,6$ & 4,5 & 0,2 & 0,9 & $-1,5$ & 1,8 & 0,5 & 1,3 \\
\hline Abduction & 0,997 & 0,994 & 0,999 & 1,6 & $-3,7$ & 9,7 & 0,1 & 0,3 & $-0,6$ & 0,7 & 0,2 & 0,5 \\
\hline Adduction & 0,996 & 0,990 & 0,998 & $-3,9$ & $-9,2$ & 3,5 & 0,0 & 0,5 & $-1,0$ & 0,9 & 0,2 & 0,6 \\
\hline Lateral rotation & 0,993 & 0,983 & 0,997 & $-15,8$ & $-27,0$ & $-4,8$ & $-0,1$ & 1,1 & $-2,3$ & 2,2 & 0,6 & 1,5 \\
\hline Medial rotation & 0,992 & 0,981 & 0,997 & $-7,4$ & $-22,4$ & 5,9 & 0,4 & 1,3 & $-2,2$ & 2,7 & 0,7 & 1,9 \\
\hline \multicolumn{13}{|l|}{$6^{\circ}$ Insole (Peak) } \\
\hline Flexion & 0,924 & 0,822 & 0,968 & 43,6 & 35,8 & 49,4 & $-0,1$ & 2,0 & $-4,0$ & 4,0 & 1,0 & 2,8 \\
\hline Extension & 0,948 & 0,876 & 0,978 & $-1,4$ & $-7,4$ & 5,3 & $-0,1$ & 1,4 & $-2,8$ & 2,8 & 0,7 & 2,0 \\
\hline Abduction & 0,999 & 0,997 & 0,999 & 1,7 & $-3,9$ & 9,9 & 0,0 & 0,2 & $-0,4$ & 0,5 & 0,1 & 0,3 \\
\hline Adduction & 0,988 & 0,972 & 0,995 & $-3,7$ & $-9,4$ & 3,4 & 0,1 & 0,7 & $-1,3$ & 1,4 & 0,4 & 1,0 \\
\hline Lateral rotation & 0,996 & 0,991 & 0,998 & $-15,9$ & $-29,5$ & $-5,7$ & 0,1 & 0,8 & $-1,5$ & 1,6 & 0,4 & 1,1 \\
\hline Medial rotation & 0,995 & 0,988 & 0,998 & $-7,5$ & $-24,1$ & 5,0 & $-0,1$ & 1,1 & $-2,2$ & 2,1 & 0,5 & 1,5 \\
\hline \multicolumn{13}{|l|}{$8^{\circ}$ Insole (Peak) } \\
\hline Flexion & 0,959 & 0,904 & 0,983 & 43,7 & 36,5 & 48,8 & $-0,1$ & 1,5 & $-3,0$ & 2,9 & 0,7 & 2,0 \\
\hline Extension & 0,957 & 0,899 & 0,982 & $-1,1$ & $-8,0$ & 6,3 & $-0,2$ & 1,3 & $-2,7$ & 2,6 & 0,7 & 1,8 \\
\hline Abduction & 0,996 & 0,992 & 0,998 & 1,7 & $-3,6$ & 10,4 & $-0,1$ & 0,4 & $-0,8$ & 0,8 & 0,2 & 0,5 \\
\hline Adduction & 0,994 & 0,985 & 0,997 & $-3,8$ & $-8,9$ & 3,5 & 0,1 & 0,5 & $-1,0$ & 1,1 & 0,3 & 0,8 \\
\hline Lateral rotation & 0,991 & 0,980 & 0,996 & $-16,0$ & $-30,2$ & $-6,4$ & 0,2 & 1,2 & $-2,2$ & 2,4 & 0,6 & 1,7 \\
\hline Medial rotation & 0,997 & 0,994 & 0,999 & $-7,3$ & $-23,5$ & 5,0 & $-0,3$ & 0,8 & $-1,8$ & 1,5 & 0,4 & 1,1 \\
\hline \multicolumn{13}{|l|}{$10^{\circ}$ Insole (Peak) } \\
\hline Flexion & 0,970 & 0,930 & 0,987 & 43,9 & 36,1 & 49,2 & 0,1 & 1,4 & $-2,5$ & 2,7 & 0,7 & 1,9 \\
\hline Extension & 0,952 & 0,886 & 0,980 & $-1,3$ & $-8,6$ & 5,6 & 0,6 & 1,4 & $-2,1$ & 2,7 & 0,7 & 1,9 \\
\hline Abduction & 0,997 & 0,994 & 0,999 & 1,7 & $-3,7$ & 9,7 & 0,0 & 0,3 & $-0,7$ & 0,6 & 0,2 & 0,4 \\
\hline Adduction & 0,995 & 0,987 & 0,998 & $-3,8$ & $-9,0$ & 3,4 & 0,1 & 0,5 & $-0,9$ & 1,0 & 0,2 & 0,7 \\
\hline Lateral rotation & 0,991 & 0,979 & 0,996 & $-15,6$ & $-29,3$ & $-5,2$ & 0,2 & 1,3 & $-2,3$ & 2,5 & 0,6 & 1,7 \\
\hline Medial rotation & 0,992 & 0,982 & 0,997 & $-7,5$ & $-24,4$ & 4,7 & 0,1 & 1,3 & $-2,4$ & 2,5 & 0,6 & 1,7 \\
\hline
\end{tabular}

ICC, intraclass correlation coefficient; 95\% CI, 95\% confidence interval for the ICC; Mean, mean of two sets of trials; Minimum and Maximum, minimum and maximum value of trials; Diff, mean of the differences between set 1 minus set 2; SDdiff, standard deviation of the differences; 95\% LL and UL, Bland and Altman 95\% limits of agreement; SEM, standard error of measurement; MDC, minimal detectable change; Kinematic values: $\mathrm{X}(+=$ flexion $/-=$ extension), $\mathrm{Y}(+=$ abduction $/-=$ adduction $) ; \mathrm{Z}(+=$ medial rotation/ $-=$ lateral rotation). 
Ferreira et al., Physical Therapy and Rehabilitation 2020,

http://www.hoajonline.com/journals/pdf/2055-2386-7-10.pdf

doi: $10.7243 / 2055-2386-7-10$

Table 2. Reliability values for kinetic parameters for different insoles: knee peak joint moment $(\mathrm{Nm} / \mathrm{kg})$.

\begin{tabular}{|c|c|c|c|c|c|c|c|c|c|c|c|c|}
\hline Kinetic (Nm/kg) & ICC & $95 \% \mathrm{CI}$ & & Mean & Minimum & Maximum & Diff & $\mathrm{SD}_{\text {diff }}$ & 95\% LL & 95\% UL & SEM & MDC \\
\hline \multicolumn{13}{|l|}{$0^{\circ}$ Insole (Peak) } \\
\hline Flexion & 0,977 & 0,947 & 0,990 & 0,612 & 0,147 & 1,130 & $-0,016$ & 0,075 & $-0,163$ & 0,147 & 0,037 & 0,103 \\
\hline Extension & 0,833 & 0,607 & 0,929 & $-0,270$ & $-0,418$ & $-0,173$ & 0,019 & 0,043 & $-0,066$ & 0,095 & 0,027 & 0,074 \\
\hline Abduction & 0,934 & 0,844 & 0,972 & $-0,064$ & $-0,144$ & $-0,028$ & $-0,005$ & 0,016 & $-0,036$ & 0,031 & 0,008 & 0,022 \\
\hline Adduction & 0,986 & 0,968 & 0,994 & 0,424 & 0,205 & 0,690 & $-0,007$ & 0,029 & $-0,064$ & 0,056 & 0,014 & 0,039 \\
\hline Lateral rotation & 0,870 & 0,694 & 0,945 & $-0,129$ & $-0,191$ & $-0,053$ & 0,002 & 0,024 & $-0,046$ & 0,050 & 0,013 & 0,036 \\
\hline Medial rotation & 0,978 & 0,949 & 0,991 & 0,106 & 0,008 & 0,253 & $-0,002$ & 0,017 & $-0,035$ & 0,034 & 0,009 & 0,025 \\
\hline \multicolumn{13}{|l|}{$2^{\circ}$ Insole (Peak) } \\
\hline Flexion & 0,967 & 0,922 & 0,986 & 0,620 & 0,181 & 1,073 & $-0,016$ & 0,087 & $-0,187$ & 0,174 & 0,044 & 0,122 \\
\hline Extension & 0,907 & 0,781 & 0,961 & $-0,277$ & $-0,412$ & $-0,178$ & 0,019 & 0,038 & $-0,056$ & 0,077 & 0,020 & 0,055 \\
\hline Abduction & 0,828 & 0,593 & 0,927 & $-0,056$ & $-0,140$ & $-0,022$ & $-0,003$ & 0,021 & $-0,045$ & 0,044 & 0,011 & 0,032 \\
\hline Adduction & 0,985 & 0,964 & 0,994 & 0,427 & 0,243 & 0,698 & 0,012 & 0,028 & $-0,042$ & 0,055 & 0,014 & 0,038 \\
\hline Lateral rotation & 0,903 & 0,771 & 0,959 & $-0,127$ & $-0,185$ & $-0,038$ & 0,003 & 0,020 & $-0,037$ & 0,040 & 0,010 & 0,029 \\
\hline Medial rotation & 0,986 & 0,966 & 0,994 & 0,109 & 0,004 & 0,264 & 0,002 & 0,016 & $-0,029$ & 0,031 & 0,008 & 0,022 \\
\hline \multicolumn{13}{|l|}{$4^{\circ}$ Insole (Peak) } \\
\hline Flexion & 0,974 & 0,938 & 0,989 & 0,587 & 0,187 & 1,131 & $-0,004$ & 0,075 & $-0,151$ & 0,148 & 0,037 & 0,104 \\
\hline Extension & 0,900 & 0,764 & 0,958 & $-0,280$ & $-0,422$ & $-0,149$ & $-0,002$ & 0,040 & $-0,080$ & 0,080 & 0,020 & 0,057 \\
\hline Abduction & 0,540 & $-0,086$ & 0,805 & $-0,055$ & $-0,100$ & $-0,019$ & 0,004 & 0,031 & $-0,056$ & 0,067 & 0,018 & 0,051 \\
\hline Adduction & 0,981 & 0,955 & 0,992 & 0,410 & 0,226 & 0,695 & 0,006 & 0,032 & $-0,057$ & 0,064 & 0,016 & 0,045 \\
\hline Lateral rotation & 0,936 & 0,849 & 0,973 & $-0,123$ & $-0,179$ & $-0,055$ & $-0,007$ & 0,016 & $-0,038$ & 0,032 & 0,008 & 0,023 \\
\hline Medial rotation & 0,982 & 0,957 & 0,992 & 0,105 & 0,008 & 0,274 & $-0,004$ & 0,016 & $-0,036$ & 0,032 & 0,008 & 0,022 \\
\hline \multicolumn{13}{|l|}{$6^{\circ}$ Insole (Peak) } \\
\hline Flexion & 0,980 & 0,954 & 0,992 & 0,603 & 0,221 & 1,103 & $-0,004$ & 0,062 & $-0,125$ & 0,122 & 0,031 & 0,085 \\
\hline Extension & 0,787 & 0,497 & 0,910 & $-0,283$ & $-0,442$ & $-0,158$ & $-0,003$ & 0,058 & $-0,117$ & 0,120 & 0,032 & 0,088 \\
\hline Abduction & 0,890 & 0,742 & 0,954 & $-0,058$ & $-0,137$ & $-0,037$ & $-0,004$ & 0,017 & $-0,039$ & 0,035 & 0,009 & 0,025 \\
\hline Adduction & 0,985 & 0,965 & 0,994 & 0,412 & 0,235 & 0,674 & $-0,004$ & 0,027 & $-0,057$ & 0,054 & 0,014 & 0,038 \\
\hline Lateral rotation & 0,904 & 0,774 & 0,959 & $-0,129$ & $-0,200$ & $-0,078$ & 0,003 & 0,019 & $-0,035$ & 0,039 & 0,010 & 0,028 \\
\hline Medial rotation & 0,977 & 0,945 & 0,990 & 0,107 & 0,001 & 0,261 & 0,001 & 0,019 & $-0,036$ & 0,037 & 0,009 & 0,026 \\
\hline \multicolumn{13}{|l|}{$8^{\circ}$ Insole (Peak) } \\
\hline Flexion & 0,980 & 0,953 & 0,991 & 0,638 & 0,195 & 1,295 & 0,009 & 0,072 & $-0,131$ & 0,142 & 0,036 & 0,099 \\
\hline Extension & 0,855 & 0,658 & 0,939 & $-0,284$ & $-0,485$ & $-0,196$ & 0,008 & 0,048 & $-0,087$ & 0,098 & 0,025 & 0,071 \\
\hline Abduction & 0,923 & 0,818 & 0,967 & $-0,054$ & $-0,131$ & $-0,020$ & $-0,005$ & 0,016 & $-0,036$ & 0,032 & 0,008 & 0,023 \\
\hline Adduction & 0,996 & 0,990 & 0,998 & 0,408 & 0,229 & 0,690 & 0,001 & 0,015 & $-0,029$ & 0,030 & 0,008 & 0,021 \\
\hline Lateral rotation & 0,898 & 0,760 & 0,957 & $-0,130$ & $-0,197$ & $-0,055$ & 0,008 & 0,024 & $-0,040$ & 0,049 & 0,013 & 0,035 \\
\hline Medial rotation & 0,976 & 0,944 & 0,990 & 0,111 & 0,011 & 0,271 & 0,002 & 0,020 & $-0,037$ & 0,039 & 0,010 & 0,027 \\
\hline \multicolumn{13}{|l|}{$10^{\circ}$ Insole (Peak) } \\
\hline Flexion & 0,969 & 0,927 & 0,987 & 0,600 & 0,170 & 1,017 & 0,000 & 0,077 & $-0,152$ & 0,153 & 0,039 & 0,108 \\
\hline Extension & 0,883 & 0,723 & 0,950 & $-0,272$ & $-0,459$ & $-0,144$ & 0,005 & 0,044 & $-0,082$ & 0,089 & 0,023 & 0,064 \\
\hline Abduction & 0,579 & 0,007 & 0,821 & $-0,052$ & $-0,096$ & $-0,021$ & $-0,003$ & 0,025 & $-0,052$ & 0,054 & 0,015 & 0,041 \\
\hline Adduction & 0,985 & 0,964 & 0,994 & 0,402 & 0,189 & 0,668 & 0,003 & 0,029 & $-0,055$ & 0,058 & 0,015 & 0,041 \\
\hline Lateral rotation & 0,855 & 0,659 & 0,939 & $-0,123$ & $-0,188$ & $-0,052$ & 0,008 & 0,026 & $-0,044$ & 0,054 & 0,014 & 0,039 \\
\hline Medial rotation & 0,969 & 0,926 & 0,987 & 0,106 & 0,011 & 0,255 & 0,000 & 0,021 & $-0,042$ & 0,042 & 0,011 & 0,029 \\
\hline
\end{tabular}

ICC. intraclass correlation coefficient; 95\% CI. 95\% confidence interval for the ICC; mean of two sets of trials; Minimum and Maximum, minimum and maximum value of trials; Diff, mean of the differences between set 1 minus set 2; SDdiff. standard deviation of the differences; 95\% LL and UL. Bland and Altman 95\% limits of agreement; SEM. standard error of measurement; MDC. minimal detectable change; Kinetic values: $\mathrm{X}(+=$ flexion $/-=$ extension), $\mathrm{Y}(+=$ adduction $/-=$ abduction $) ; \mathrm{Z}(+=$ medial rotation/ $-=$ lateral rotation) 
Ferreira et al., Physical Therapy and Rehabilitation 2020,

http://www.hoajonline.com/journals/pdf/2055-2386-7-10.pdf

doi: 10.7243/2055-2386-7-10

Table 3. Main EKAM parameters for different insoles and average insoles: first (1st) and second ( 2 nd) peak EKAM and KAAI.

\begin{tabular}{|c|c|c|c|c|c|c|c|c|c|c|c|c|}
\hline \multirow{2}{*}{$\begin{array}{l}\text { Kinetic (Nm/Kg) } \\
0^{\circ} \text { Insole (Peak) }\end{array}$} & \multirow[t]{2}{*}{ ICC } & \multicolumn{2}{|c|}{ 95\% CI } & \multirow[t]{2}{*}{ Mean } & \multirow[t]{2}{*}{ Minimum } & \multirow[t]{2}{*}{ Maximum } & \multirow[t]{2}{*}{ Diff } & \multirow[t]{2}{*}{$\mathrm{SD}_{\text {diff }}$} & \multirow[t]{2}{*}{ 95\% LL } & \multirow[t]{2}{*}{ 95\% UL } & \multirow[t]{2}{*}{ SEM } & \multirow[t]{2}{*}{ MDC } \\
\hline & & & & & & & & & & & & \\
\hline $1^{\text {st }}$ Peak EKAM & 0,987 & 0,969 & 0,995 & 0,399 & 0,157 & 0,690 & $-0,011$ & 0,030 & $-0,070$ & 0,058 & 0,015 & 0,040 \\
\hline $2^{\text {nd }}$ Peak EKAM & 0,985 & 0,964 & 0,994 & 0,339 & 0,122 & 0,573 & $-0,003$ & 0,033 & $-0,067$ & 0,063 & 0,016 & 0,043 \\
\hline KAAI $\left(\mathrm{Nm} / \mathrm{kg}^{\star} \mathrm{s}\right)$ & 0,987 & 0,969 & 0,994 & 0,140 & 0,038 & 0,245 & 0,000 & 0,015 & $-0,029$ & 0,029 & 0,007 & 0,020 \\
\hline \multicolumn{13}{|l|}{$2^{\circ}$ Insole (Peak) } \\
\hline $1^{\text {st }}$ Peak EKAM & 0,989 & 0,973 & 0,995 & 0,403 & 0,130 & 0,698 & 0,009 & 0,027 & $-0,044$ & 0,053 & 0,013 & 0,037 \\
\hline $2^{\text {nd }}$ Peak EKAM & 0,986 & 0,967 & 0,994 & 0,344 & 0,107 & 0,559 & 0,006 & 0,027 & $-0,048$ & 0,054 & 0,014 & 0,038 \\
\hline KAAI $\left(\mathrm{Nm} / \mathrm{kg}^{\star} \mathrm{s}\right)$ & 0,992 & 0,981 & 0,997 & 0,142 & 0,033 & 0,232 & 0,003 & 0,010 & $-0,017$ & 0,021 & 0,005 & 0,014 \\
\hline \multicolumn{13}{|l|}{$4^{\circ}$ Insole (Peak) } \\
\hline $1^{\text {st }}$ Peak EKAM & 0,982 & 0,958 & 0,992 & 0,378 & 0,113 & 0,695 & 0,005 & 0,035 & $-0,063$ & 0,069 & 0,017 & 0,048 \\
\hline $2^{\text {nd }}$ Peak EKAM & 0,984 & 0,962 & 0,993 & 0,339 & 0,132 & 0,544 & 0,007 & 0,030 & $-0,052$ & 0,060 & 0,015 & 0,042 \\
\hline KAAI (Nm/kg*s) & 0,985 & 0,965 & 0,994 & 0,137 & 0,040 & 0,225 & 0,004 & 0,014 & $-0,024$ & 0,028 & 0,007 & 0,020 \\
\hline \multicolumn{13}{|l|}{$6^{\circ}$ Insole (Peak) } \\
\hline $1^{\text {st }}$ Peak EKAM & 0,991 & 0,978 & 0,996 & 0,385 & 0,121 & 0,674 & 0,001 & 0,025 & $-0,049$ & 0,050 & 0,013 & 0,035 \\
\hline $2^{\text {nd }}$ Peak EKAM & 0,987 & 0,969 & 0,994 & 0,340 & 0,155 & 0,525 & $-0,006$ & 0,025 & $-0,055$ & 0,050 & 0,013 & 0,035 \\
\hline KAAI (Nm/kg*s) & 0,993 & 0,983 & 0,997 & 0,138 & 0,039 & 0,236 & $-0,002$ & 0,010 & $-0,021$ & 0,019 & 0,005 & 0,014 \\
\hline \multicolumn{13}{|l|}{$8^{\circ}$ Insole (Peak) } \\
\hline $1^{\text {st }}$ Peak EKAM & 0,994 & 0,987 & 0,998 & 0,373 & 0,108 & 0,690 & $-0,002$ & 0,020 & $-0,041$ & 0,039 & 0,010 & 0,027 \\
\hline $2^{\text {nd }}$ Peak EKAM & 0,992 & 0,982 & 0,997 & 0,348 & 0,120 & 0,568 & 0,001 & 0,021 & $-0,040$ & 0,041 & 0,010 & 0,028 \\
\hline KAAI (Nm/kg*s) & 0,992 & 0,982 & 0,997 & 0,138 & 0,030 & 0,231 & $-0,002$ & 0,011 & $-0,023$ & 0,021 & 0,005 & 0,015 \\
\hline \multicolumn{13}{|l|}{$10^{\circ}$ Insole (Peak) } \\
\hline $1^{\text {st }}$ Peak EKAM & 0,988 & 0,971 & 0,995 & 0,356 & 0,086 & 0,668 & 0,001 & 0,030 & $-0,057$ & 0,059 & 0,015 & 0,041 \\
\hline $2^{\text {nd }}$ Peak EKAM & 0,978 & 0,949 & 0,991 & 0,345 & 0,111 & 0,576 & 0,006 & 0,034 & $-0,062$ & 0,068 & 0,017 & 0,048 \\
\hline KAAI (Nm/kg*s) & 0,983 & 0,960 & 0,993 & 0,133 & 0,029 & 0,224 & 0,000 & 0,015 & $-0,029$ & 0,029 & 0,007 & 0,021 \\
\hline \multicolumn{13}{|l|}{ Average (Peak) } \\
\hline $1^{\text {st }}$ Peak EKAM & 0,988 & 0,983 & 0,991 & 0,382 & 0,086 & 0,698 & 0,001 & 0,028 & $-0,055$ & 0,056 & 0,014 & 0,039 \\
\hline $2^{\text {nd }}$ Peak EKAM & 0,985 & 0,978 & 0,989 & 0,342 & 0,107 & 0,576 & 0,002 & 0,029 & $-0,054$ & 0,058 & 0,014 & 0,039 \\
\hline KAAI $\left(\mathrm{Nm} / \mathrm{kg}^{\star} \mathrm{s}\right)$ & 0,988 & 0,983 & 0,991 & 0,138 & 0,029 & 0,245 & 0,001 & 0,013 & $-0,024$ & 0,025 & 0,006 & 0,018 \\
\hline
\end{tabular}

ICC. intraclass correlation coefficient; 95\% CI. 95\% confidence interval for the ICC; mean of two sets of trials; Minimum and Maximum, minimum and maximum value of trials; Diff, mean of the differences between set 1 minus set 2; SDdiff. standard deviation of the differences; 95\% LL and UL. Bland and Altman 95\% limits of agreement; SEM. standard error of measurement; MDC. minimal detectable change.
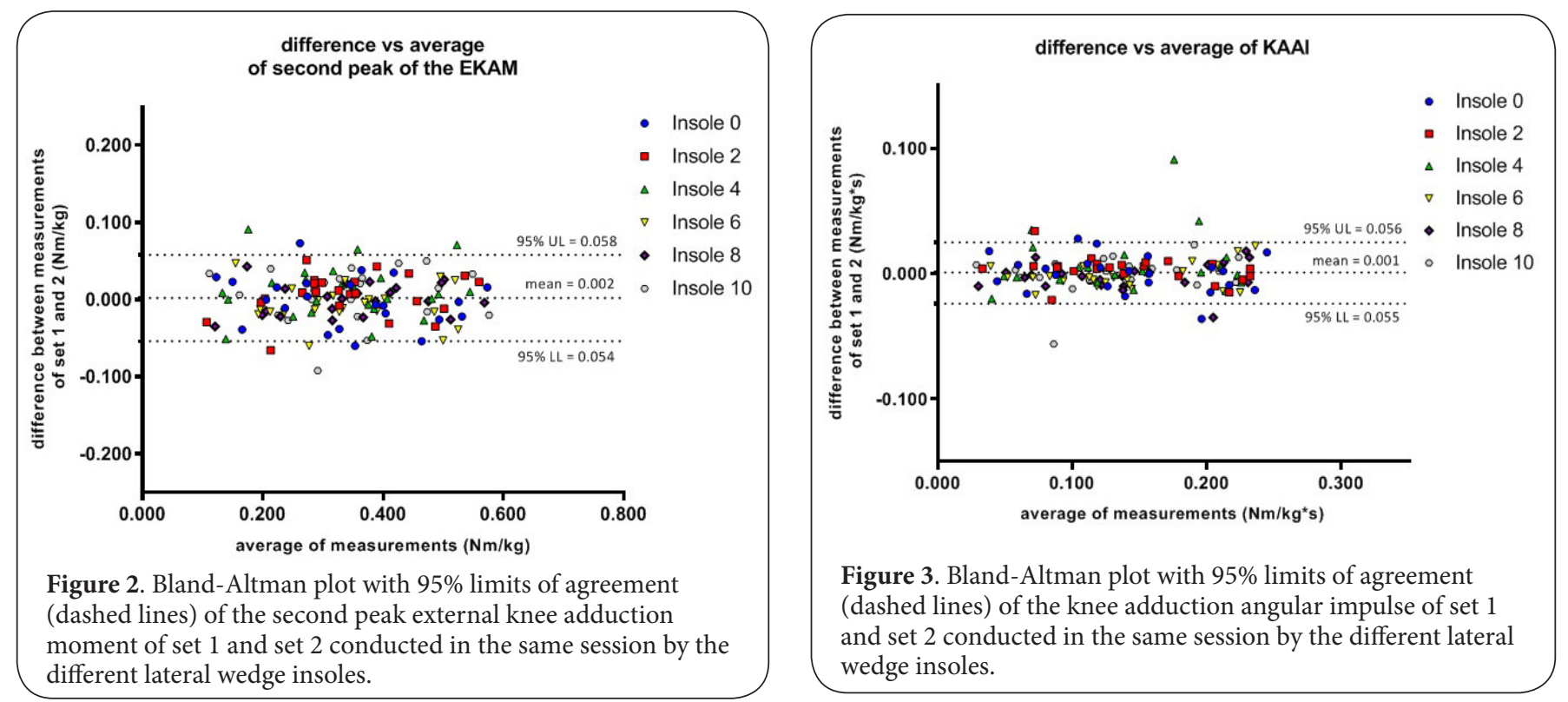
also reported similar values with an ICC from 0.47 to 0.84 . The SEM in our study was less than $1.0^{\circ}$, slightly lower than in previous studies. A greater difference was found in MDC. In our study, we found outcomes less than $2.9^{\circ}$ which compares with results of about $4.7^{\circ}$ in the Wilken et al. study and greater than $4.0^{\circ}$ in the Meldrum et al. study. For kinetic parameters, and those relevant to the EKAM, we also found results with excellent consistency, higher than those reported in previous studies. For instance, for the first peak of the EKAM, we found an ICC $=0.988$ while in the study of Wilken et al. [24] an ICC= 0.76 . These results enhanced than those reported in previous

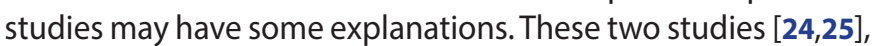
used different motion capture systems and markers sets which makes it challenging to compare with our study. The previous studies used a full body and a Helen Hays set respectively. In our study, we used a 42-passive marker set just in the pelvis and the lower limbs. This redundancy of passive markers on the lower limbs may allow a better consistency in particular on the transverse plane, which is usually less reliable [15]. An important aspect to keep in mind is that in previous studies the design of the study was a test-retest design with differences in days between the two test sessions. As the objective of our study was to study repeatability within-session, the results are obviously more consistent by reducing the marker placement error. However, for an adjusted prescription of wedge insoles based on biomechanical acute effects, the most important issue in our view was to assure that we could distinguish differences in repeatability of kinematic and kinetic data of different wedge angles within a subject.

Some studies have shown that increased EKAM has been related to osteoarthritis severity and disease progression $[26,27]$. Several types of approaches to reduce EKAM have already been tested [28-30]. However, it was not possible to reach consensus on the best approach. Most studies use a single angulation as a strategy $[5,13,31]$. Some, applied two types of insoles in the procedures $[9,32]$ and a limited number use an approach based on the best biomechanical fit $[3,33]$. Those who did so, however were based on other parameters than the biomechanical parameters, such as the feeling of discomfort or pain reduction $[3,34]$. If we individualized the \% of change in the first peak of the EKAM, like as in the Hinman study [11], we found that of 73 individuals, 16 showed an increase of the EKAM. Those who presented reduction, some presented a reduction close to $25 \%$ while others a reduction of less than $5 \%$. The results of our study suggest that the assessment with different wedge angulations can be a more accurate approach to propose lateral wedge insoles. "Different insoles for different patients", is a way that may be more virtuous than the current one. In a recent study [13], a biomechanical approach was performed, but only to remove the non-biomechanical responders. A previous study also tested the effects of different wedge angles on EKAM. However, to our best knowledge it was not possible to find studies about reliability of the measurement. The results of our study showed that the procedures used to collect data allow very good repeatability of the trials.

Results of this study must be read considering some limitations. The first and most important limitation is that subjects in this study were young and without medial knee OA. Individuals suffering from knee osteoarthritis generally show marked structural changes, which does not allow compare the response to the different wedge insoles. Second, only one researcher conducted the experiment, therefore the intertester reliability was not contemplated being this an important aspect in laboratories where subjects are usually tested by different testers. It has been shown that inter tester reliability is lower than that of intra tester [15]. Finally, the sample was a convenience sample, which limits generalization.

\section{Conclusion}

The present results support the use and facilitate the interpretation of EKAM parameters for prescription of lateral wedge insoles. The use of error estimates in clinical gait analysis may improve the accuracy of clinical interpretation particularly after the application of treatments. In addition, we believe that this study provides preliminary data, which may be useful for clinical interpretation and decision to recommend the insole that best fits to the condition of the patient with medial knee osteoarthritis.

\section{Competing interests}

The authors declare that they have no competing interests.

\section{Authors' contributions}

\begin{tabular}{|l|c|c|c|c|}
\hline Authors' contributions & VF & LM & RS & PR \\
\hline Research concept and design & $\checkmark$ & $\checkmark$ & $\checkmark$ & $\checkmark$ \\
\hline Collection and/or assembly of data & $\checkmark$ & -- & $\checkmark$ & -- \\
\hline Data analysis and interpretation & $\checkmark$ & $\checkmark$ & $\checkmark$ & $\checkmark$ \\
\hline Writing the article & $\checkmark$ & $\checkmark$ & $\checkmark$ & $\checkmark$ \\
\hline Critical revision of the article & $\checkmark$ & $\checkmark$ & $\checkmark$ & $\checkmark$ \\
\hline Final approval of article & $\checkmark$ & $\checkmark$ & $\checkmark$ & $\checkmark$ \\
\hline Statistical analysis & $\checkmark$ & -- & $\checkmark$ & -- \\
\hline
\end{tabular}

Publication history

Editor: Gordon John Alderink, Grand Valley State University, USA. Received: 13-July-2020 Final Revised: 30-Aug-2020

Accepted: 11-Sept-2020 Published: 22-Sept-2020

\section{References}

1. McAlindon, T.E., et al., OARSI guidelines for the non-surgical management of knee osteoarthritis. Osteoarthritis Cartilage, 2014. 22(3): p. 363-88. https://www.ncbi.nlm.nih.gov/pubmed/24462672

2. Hinman, R.S., et al., Lateral wedges in knee osteoarthritis: what are their immediate clinical and biomechanical effects and can these predict a three-month clinical outcome? Arthritis Rheum, 2008. 59(3): p. 408-15. https://www.ncbi.nlm.nih.gov/pubmed/18311763

3. Butler, R.J., et al., The effect of a subject-specific amount of lateral wedge on knee mechanics in patients with medial knee osteoarthritis. J Orthop Res, 2007. 25(9): p. 1121-7. https://www.ncbi.nlm.nih.gov/ pubmed/17469197

4. Kerrigan, D.C., et al., Effectiveness of a lateral-wedge insole on knee varus 
Ferreira et al., Physical Therapy and Rehabilitation 2020,

http://www.hoajonline.com/journals/pdf/2055-2386-7-10.pdf

doi: $10.7243 / 2055-2386-7-10$

torque in patients with knee osteoarthritis. Arch Phys Med Rehabil, 2002. 83(7): p. 889-93. http://www.ncbi.nlm.nih.gov/pubmed/12098144

5. Fantini Pagani, C.H., M. Hinrichs, and G.P. Bruggemann, Kinetic and kinematic changes with the use of valgus knee brace and lateral wedge insoles in patients with medial knee osteoarthritis. J Orthop Res, 2012. 30(7): p. 1125-32. http://www.ncbi.nlm.nih.gov/pubmed/22161816

6. Maly, M.R., E.G. Culham, and P.A. Costigan, Static and dynamic biomechanics of foot orthoses in people with medial compartment knee osteoarthritis. Clin Biomech (Bristol, Avon), 2002. 17(8): p. 603-10. https://www.ncbi.nlm.nih.gov/pubmed/12243720

7. Kakihana, W., et al., Effects of laterally wedged insoles on knee and subtalar joint moments. Arch Phys Med Rehabil, 2005. 86(7): p. 1465-71. https://www.ncbi.nlm.nih.gov/pubmed/16003682

8. Dessery, Y., et al., Effects of foot orthoses with medial arch support and lateral wedge on knee adduction moment in patients with medial knee osteoarthritis. Prosthet Orthot Int, 2016. https://www.ncbi.nlm.nih.gov/ pubmed/27555447

9. Abdallah, A.A. and A.Y. Radwan, Biomechanical changes accompanying unilateral and bilateral use of laterally wedged insoles with medial arch supports in patients with medial knee osteoarthritis. Clin Biomech, 2011. 26(7): p. 783-9. https://www.ncbi.nlm.nih.gov/pubmed/21497965

10. Ferreira, V., et al., The optimal degree of lateral wedge insoles for reducing knee joint load: a systematic review and meta-analysis. Archives of Physiotherapy, 2019. 9(1): p. 18. https://doi.org/10.1186/s40945-0190068-1

11. Hinman, R.S., et al., Lateral wedge insoles for medial knee osteoarthritis: effects on lower limb frontal plane biomechanics. Clin Biomech (Bristol, Avon), 2012. 27(1): p. 27-33. https://www.ncbi.nlm.nih.gov/ pubmed/21862189

12. Jones, R.K., et al., The relationship between reductions in knee loading and immediate pain response whilst wearing lateral wedged insoles in knee osteoarthritis. J Orthop Res, 2014. 32(9): p. 1147-54. https://www. ncbi.nlm.nih.gov/pubmed/24903067

13. Lewinson, R.T., et al., Reduced knee adduction moments for management of knee osteoarthritis:: A three month phase I/II randomized controlled trial. Gait Posture, 2016. 50: p. 60-68. https://www.ncbi.nlm.nih.gov/ pubmed $/ 27580080$

14. Schwartz, M.H., J.P. Trost, and R.A. Wervey, Measurement and management of errors in quantitative gait data. Gait Posture, 2004. 20(2): p. 196-203. https://www.ncbi.nlm.nih.gov/pubmed/15336291

15. McGinley, J.L., et al., The reliability of three-dimensional kinematic gait measurements: a systematic review. Gait Posture, 2009. 29(3): p. 360-9. https://www.ncbi.nlm.nih.gov/pubmed/19013070

16. Godwin, M., et al., Pragmatic controlled clinical trials in primary care: the struggle between external and internal validity. BMC Med Res Methodol, 2003. 3: p. 28. https://www.ncbi.nlm.nih.gov/pubmed/14690550

17. Kottner, J., et al., Guidelines for Reporting Reliability and Agreement Studies (GRRAS) were proposed. J Clin Epidemiol, 2011. 64(1): p. 96-106. https://www.ncbi.nlm.nih.gov/pubmed/21130355

18. Bartlett, J.W. and C. Frost, Reliability, repeatability and reproducibility: analysis of measurement errors in continuous variables. Ultrasound Obstet Gynecol, 2008. 31(4): p. 466-75. https://www.ncbi.nlm.nih.gov/ pubmed/18306169

19. de Vet, H.C., et al., When to use agreement versus reliability measures. J Clin Epidemiol, 2006. 59(10): p. 1033-9. http://www.ncbi.nlm.nih.gov/ pubmed/16980142

20. de Vet, H.C., et al., Minimal changes in health status questionnaires: distinction between minimally detectable change and minimally important change. Health Qual Life Outcomes, 2006. 4: p. 54. https:// www.ncbi.nlm.nih.gov/pubmed/16925807

21. Cappozzo, A., et al., Position and orientation in space of bones during movement: anatomical frame definition and determination. Clin Biomech (Bristol, Avon), 1995. 10(4): p. 171-178. http://www.ncbi.nlm.nih.gov/ pubmed/11415549

22. Kinds, M.B., et al., Evaluation of separate quantitative radiographic features adds to the prediction of incident radiographic osteoarthritis in individuals with recent onset of knee pain: 5-year follow-up in the CHECK cohort. Osteoarthritis Cartilage, 2012. 20(6): p. 548-56. https://www. ncbi.nlm.nih.gov/pubmed/22366685

23. Morphett, A.L., C.M. Crawford, and D. Lee, The use of electromagnetic tracking technology for measurement of passive cervical range of motion: a pilot study. Journal of Manipulative and Physiological Therapeutics, 2003. 26(3): p. 152-159. http://www.sciencedirect.com/science/article/ pii/S0161475402541075

24. Wilken, J.M., et al., Reliability and Minimal Detectible Change values for gait kinematics and kinetics in healthy adults. Gait Posture, 2012. 35(2): p. 301-7. https://www.ncbi.nlm.nih.gov/pubmed/22041096

25. Meldrum, D., et al., Test-retest reliability of three dimensional gait analysis: including a novel approach to visualising agreement of gait cycle waveforms with Bland and Altman plots. Gait Posture, 2014. 39(1): p. 265-71. https://www.ncbi.nlm.nih.gov/pubmed/24139682

26. Miyazaki, T., et al., Dynamic load at baseline can predict radiographic disease progression in medial compartment knee osteoarthritis. Ann Rheum Dis, 2002. 61(7): p. 617-22. https://www.ncbi.nlm.nih.gov/ pubmed/12079903

27. Hall, M., et al., The knee adduction moment and knee osteoarthritis symptoms: relationships according to radiographic disease severity. Osteoarthritis Cartilage, 2016. https://www.ncbi.nlm.nih.gov/ pubmed/27616685

28. Simic, M., et al., Gait modification strategies for altering medial knee joint load: a systematic review. Arthritis Care Res (Hoboken), 2011. 63(3): p. 405-26. http://www.ncbi.nlm.nih.gov/pubmed/20981808

29. Moyer, R.F., et al., Biomechanical effects of valgus knee bracing: a systematic review and meta-analysis. Osteoarthritis Cartilage, 2015. 23(2): p. 178-88. https://www.ncbi.nlm.nih.gov/pubmed/25447975

30. Ferreira, G.E., et al., The effect of exercise therapy on knee adduction moment in individuals with knee osteoarthritis: A systematic review. Clin Biomech (Bristol, Avon), 2015. 30(6): p. 521-7. https://www.ncbi.nlm.nih. gov/pubmed $/ 25896448$

31. Hinman, R.S., K.A. Bowles, and K.L. Bennell, Laterally wedged insoles in knee osteoarthritis: do biomechanical effects decline after one month of wear? BMC Musculoskelet Disord, 2009. 10: p. 146. https://www.ncbi. nlm.nih.gov/pubmed/19939281

32. Dessery, Y., et al., Effects of foot orthoses with medial arch support and lateral wedge on knee adduction moment in patients with medial knee osteoarthritis. Prosthet Orthot Int, 2017. 41(4): p. 356-363. https://www. ncbi.nlm.nih.gov/pubmed/27555447

33. Butler, R.J., et al., Effect of laterally wedged foot orthoses on rearfoot and hip mechanics in patients with medial knee osteoarthritis. Prosthet Orthot Int, 2009. 33(2): p. 107-16. https://www.ncbi.nlm.nih.gov/ pubmed/19367514

34. Tipnis, R.A., et al., The dose-response relationship between lateral foot wedging and the reduction of knee adduction moment. Clin Biomech (Bristol, Avon), 2014. 29(9): p. 984-9. https://www.ncbi.nlm.nih.gov/ pubmed/25246374

\section{Citation:}

Ferreira V, Machado L, Simões R and Roriz P. Intra-session reliability of different angles of lateral wedge insoles in the biomechanical parameters of the knee.

Phys Ther Rehabil. 2020; 7:10.

http://dx.doi.org/10.7243/2055-2386-7-10 